\title{
La mairie de Londres et la décentralisation : entre autonomie et indépendance
}

Devolution and the Greater London Authority: Somewhere Between Autonomy and Independence

\section{Timothy Whitton}

\section{OpenEdition} Journals

Édition électronique

URL : http://journals.openedition.org/rfcb/3418

DOI : $10.4000 /$ rfcb.3418

ISSN : 2429-4373

Éditeur

CRECIB - Centre de recherche et d'études en civilisation britannique

Édition imprimée

Date de publication : 1 avril 2005

ISBN : 2-911580-20-6

ISSN : 0248-9015

Référence électronique

Timothy Whitton, «La mairie de Londres et la décentralisation : entre autonomie et indépendance », Revue Française de Civilisation Britannique [En ligne], XIII-2 | 2005, mis en ligne le 01 avril 2005, consulté le 10 avril 2020. URL : http://journals.openedition.org/rfcb/3418; DOI : https://doi.org/10.4000/rfcb. 3418

Ce document a été généré automatiquement le 10 avril 2020.

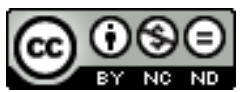

Revue française de civilisation britannique est mis à disposition selon les termes de la licence Creative Commons Attribution - Pas d'Utilisation Commerciale - Pas de Modification 4.0 International. 


\title{
La mairie de Londres et la décentralisation : entre autonomie et indépendance
}

\author{
Devolution and the Greater London Authority: Somewhere Between Autonomy \\ and Independence
}

Timothy Whitton

1 Il aura fallu attendre la deuxième élection à la nouvelle mairie de Londres pour confirmer son statut de pouvoir local « décentralisé ». La première, organisée en 2000, se devait d'accompagner la mise en place de cette nouvelle administration. Or, elle a été largement dominée par des enjeux politiques d'ordre national. Si bien que les programmes électoraux des différents candidats, leurs véritables projets pour la capitale, n'ont pas fait l'objet d'une campagne d'information digne d'une élection de cette importance. Depuis 1986, en effet, la ville n'était plus gérée par une seule autorité capable d'intervenir au-delà des frontières des différentes municipalités, mais par une multiplicité d'organismes dotés de pouvoirs et de champs d'activités mal définis. Compte tenu de cette confusion, les projets s'inspirant de la réputation de Londres en tant que capitale mondiale - l'organisation des Jeux olympiques par exemple peinaient à voir le jour. Toutefois, la mise en place d'une mairie à Londres ne répondait pas uniquement à ce besoin de disposer d'un échelon de pouvoir local pour traiter les dossiers de grande envergure. Le gouvernement central savait que si dans la capitale l'expérience se révélait positive, rien ne s'opposerait à une extension du système à d'autres villes de taille importante. À ce titre, Londres devait servir d'exemple, calqué selon certains sur le modèle américain, mais symbole, néanmoins, de la volonté affichée par le New Labour de rapprocher les citoyens de leurs élus.

2 En 2004 la mairie de Londres n'est plus, en revanche, le même symbole de la décentralisation et peut être jugée selon son bilan. La mesure phare de ce dernier est sans aucun doute la taxe "anti-embouteillages " destinée à limiter la circulation automobile dans le centre ville. L'augmentation sensible du nombre de voyages effectués grâce aux transports en commun est une conséquence directe de la taxe anti- 
embouteillages et elle est à mettre au crédit de la mairie de Londres tout comme, d'ailleurs, le réaménagement de Trafalgar Square qui, désormais, n'a rien à envier aux autres grandes places des capitales européennes. Cette transition entre pouvoir symbolique et véritable outil de gestion de la capitale doit sans doute beaucoup à Kenneth « le rouge » Livingstone, enfant terrible de la Loony Left qui tout au long des années quatre-vingt avait provoqué de sérieuses divisions au sein du Parti travailliste. Aux yeux de l'électorat britannique, la radicalisation d'une frange des travaillistes avait largement contribué à les rendre inéligibles. Une fois débarrassé de ce handicap, grâce surtout à la modernisation du parti, Tony Blair ne pouvait tolérer la présence à la tête de la capitale d'un franc-tireur capable à tout instant de défier ouvertement les instances dirigeantes de son parti. Ainsi, si le Premier ministre était disposé à permettre aux Londoniens de s'exprimer en choisissant directement leur maire, il tenait à ce que l'un de ses fidèles occupe ce poste. Mais Livingstone ne l'entendait pas ainsi et ce trublion sorti des rangs de la « vieille gauche » anglaise honnie de Tony Blair a su ravir le poste de maire de Londres au New Labour. Celui-ci l'a détesté, l'a boudé et puis l'a courtisé sachant qu'il valait mieux avoir Londres avec Livingstone confortablement installé dans le fauteuil du maire à City Hall ${ }^{2}$ que de vivre une nouvelle période de divisions, fort coûteuses en termes de crédibilité politique. C'est ainsi que Livingstone, à la tête de la municipalité de Londres, a entamé sa nouvelle période d'opposition au pouvoir central. Mais cette fois-ci ses motivations étaient différentes : si dans le premier cas l'ennemi politique se trouvait à quelques encâblures de l'autre côté de la Tamise, celui qui avait ouvertement déclaré que Livingstone serait une catastrophe pour la capitale appartient désormais au même camp que le maire de Londres. Tous les deux ont pourtant mis de l'eau dans leur vin et à la veille de l'élection de 2004, la presse titrait Blair wants Livingstone back. Au bout de quatre ans de mandat, l'heure était venue, en effet, de dresser le bilan du travail accompli par Livingstone et son équipe. Si dans l'ensemble ils obtiennent un satisfecit, ce n'est pas pour autant que les relations entre le New Labour et la mairie de Londres sont cordiales et que cette décentralisation s'est faite sans heurts.

\section{Abolir pour mieux régner ${ }^{3}$}

Il est indéniable que l'objectif du gouvernement de Margaret Thatcher, lorsqu'il décide, au début des années 1980, la dissolution des Conseils Métropolitains et surtout du Greater London Council (GLC, Conseil du Grand Londres), est de porter un coup fatal au type de "socialisme municipal» prôné surtout par la gauche la plus radicale. Livingstone et ses amis élus à l'assemblée du GLC symbolisent l'émergence de cette aile du Parti travailliste, et les conservateurs en profitent pour dénoncer à l'envi les liens indéfectibles qui existent entre ce courant radical et le travaillisme. À tel point que le Parti travailliste mettra bien des années pour se débarrasser de son image de parti inéligible, image, qui plus est, qu'il s'est en grande partie infligée lui-même. Dès le lendemain des élections municipales de 1981, qui voient Livingstone élu à la tête du GLC, Margaret Thatcher, en voyage à l'étranger, n'hésite pas à évoquer la menace qui

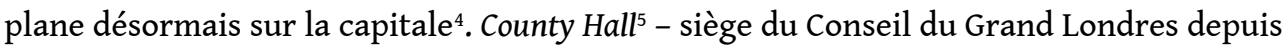
1922 - devient rapidement le fleuron du socialisme municipal dont les conservateurs entendent débarrasser le pays. Compte tenu de la faiblesse de l'Opposition au sein du Parlement, de l'autre côté de la Tamise, le groupe travailliste, mené par un Livingstone survolté, transforme County Hall en un formidable bastion anti-Thatcher ${ }^{6}$. On se 
souvient, par exemple, des immenses banderoles que l'on faisait dérouler sur les façades de County Hall pour dénoncer les choix politiques des gouvernements conservateurs, ainsi que du panneau gigantesque sur le toit du bâtiment qui affichait le taux de chômage à Londres. Dans l'ensemble, les députés conservateurs sont favorables à l'abandon des Conseils Métropolitains ${ }^{7}$, et sont disposés à soutenir le gouvernement lorsqu'il prétend que ce niveau d'administration locale est trop coûteux d'une part, et d'autre part qu'il n'est pas assez responsable vis-à-vis de ses électeurs. Néanmoins, la manière choisie par le gouvernement pour mettre un terme aux Conseils Métropolitains ne fait pas l'unanimité parmi les conservateurs, loin s'en faut, et Margaret Thatcher a bien du mal à faire passer sa loi. C'est essentiellement le fait d'annuler les élections prévues pour 1985 qui constitue la pierre d'achoppement. Cette annulation impliquera forcément une période de transition pendant laquelle la capitale sera gérée, selon les souhaits du gouvernement, non pas par des élus choisis par les Londoniens, mais par des administrateurs nommés. Les débats à la Chambre des Communes sont houleux et même l'ancien Premier ministre conservateur, Edward Heath, monte au créneau pour dénoncer la stratégie de son parti. Malgré la vague de protestations, caractérisée par le slogan Say No to No Say, ainsi que la réticence des Lords à entériner cette législation, le gouvernement conservateur sort victorieux de cette période de conflit. La loi votée en juillet 1985 sur la dissolution du Conseil du Grand Londres prévoit aussi la ventilation, auprès d'une pléthore d'organisations regroupées sous la houlette du Minister for London, de toutes les missions auparavant confiées au GLC. Le "quango " qui en dit long sur les véritables motivations du gouvernement s'appelle le London Residuary Board (LRB), l'organisme censé s'occuper de tous les dossiers du GLC laissés sans responsable une fois la loi entrée en vigueur. À titre d'exemple, la gestion du patrimoine immobilier de la ville est abandonnée et revient de fait au LRB. Quelque quatorze années plus tard, c'est justement ce dossier qui incitera le Parti travailliste à mettre en place une nouvelle administration municipale pour la capitale.

\section{Décentraliser pour mieux régner ?}

Dans son manifeste électoral de 1992, le Parti travailliste parle timidement d'instaurer une administration pour gérer la capitale, sans pour autant évoquer le terme de «mairie» et encore moins celui de maire. C'est a priori l'urbanisme et la gestion du patrimoine immobilier de Londres qui suscitent une certaine inquiétude puisque aucun organisme privé n'a les moyens de définir une vraie politique du logement pour l'ensemble de l'agglomération. En 1994 les travaillistes publient un document intitulé Working Together for London dans lequel ils appellent de leurs vœux la mise en place de la Greater London Authority, c'est-à-dire une instance administrative locale capable de gérer les intérêts de toute la ville, au-delà des frontières de chaque municipalité. Deux années plus tard, leur document consultatif, A Voice for London, est publié. Ils y préconisent, une fois de plus, la création d'une nouvelle autorité à Londres mais restent vagues quant aux responsabilités - en termes de gestion de services - qui lui seraient confiées. De toute évidence, le New Labour souhaite rester très prudent étant donné que le spectre de la confrontation entre le pouvoir municipal de Londres et le gouvernement de Westminster - même si à l'époque il s'agissait de deux camps politiques diamétralement opposés - n'est jamais très loin. Cette crainte est d'autant plus fondée que, pour l'heure, le souci principal du New Labour est de continuer à 
renforcer sa crédibilité auprès de l'électorat, et il faut à tout prix éviter de réveiller les vieux démons du GLC.

5 En 1997, dans le manifeste électoral du Parti conservateur, il est surtout question des services que l'on pourra privatiser dans la capitale. Les libéraux démocrates mentionnent la possibilité d'y créer une "strategic authority " tandis que les promesses de décentralisation du New Labour concernent principalement la mise en place d'un parlement en Écosse et d'une assemblée au Pays de Galles. Mais dans la rubrique intitulée - ironie du sort - We will clean up politics, Tony Blair s'engage à étudier la possibilité de doter les grandes villes de maires élus ${ }^{8}$ :

Le pouvoir décisionnel de proximité devrait subir moins de contraintes imposées par le gouvernement central et en même temps répondre davantage de ses actes devant les populations concernées. [...] Nous encouragerons, au sein des collectivités territoriales, la mise en place d'innovations démocratiques. Cela comprendra un espace de réflexion sur la possibilité d'élire, pour administrer les grandes villes, des maires qui seront investis de pouvoirs exécutifs?.

Plus loin dans le manifeste, Londres bénéficie d'une mention spéciale mais rien ne laisse présager que le New Labour va tout mettre en œuvre pour garder le contrôle de la capitale :

Londres est la seule capitale occidentale à ne pas être administrée par une instance élue. Suite à un référendum qui confirmera le souhait des Londoniens, il y aura un nouveau contrat pour Londres qui comprendra une nouvelle autorité amenée à faire des choix stratégiques pour la capitale et un maire, tous deux élus directement. Ils défendront les besoins de la capitale et auront la charge de planifier son avenir. Il n'y aura pas de double-emploi avec le travail accompli par les différentes municipalités mais au contraire, ils assumeront la responsabilité des questions qui touchent l'ensemble de l'agglomération, le renouveau économique, l'urbanisme, le maintien de l'ordre, les transports en commun et la protection de l'environnement. Il est urgent que Londres assume la responsabilité de sa propre gestion. $[\ldots . .]^{10}$

$7 \mathrm{Au}$ contraire, l'accent est clairement mis sur la volonté de décentraliser afin de favoriser une représentation politique de proximité. Au sein du gouvernement "fantôme", le sentiment général est que Londres doit disposer d'un échelon de pouvoir digne d'une ville internationale, et surtout digne d'une capitale européenne qui veut organiser des manifestations de grande envergure, les Jeux olympiques notamment. Pour l'instant, la multitude d'organisations qui s'occupent de la capitale tantôt en-deçà des frontières des différentes municipalités, tantôt au-delà, est qualifiée de mish-mash par la presse. Celle-ci dénonce le manque de pouvoir "supra-municipal» et pointe les méthodes de gestion existantes, obscures et guère propices à promouvoir l'intérêt de l'ensemble de la capitale. À la veille des élections législatives, l'engouement de la presse pour la candidature britannique à l'organisation des Jeux de 2004 donne l'impression qu'il s'agit là d'un enjeu politique majeur. Mais le dossier olympique ne fait l'objet que d'une seule mention dans le manifeste du New Labour, et d'aucune dans ceux des autres principales formations politiques.

8 En mars 1998, après les élections législatives et la victoire historique du New Labour, un Livre blanc est publié sur Londres. On constate tout de suite le changement de ton : A Mayor and Assembly for London: the Government's Proposals for Modernising the Governance of London. Les exigences lexicales de la « troisième voie » semblent d'ores et déjà avoir pris le dessus sur les objectifs initiaux. Il s'agit, certes, de donner les moyens à la capitale d'avoir son pouvoir exécutif, et assez curieusement, compte tenu de la tournure que 
vont prendre les événements, de concentrer le pouvoir entre les mains d'une seule personne. Mais surtout, le but avoué est de "moderniser" la gestion de Londres. Autrement dit, doter la capitale d'une mairie n'est pas une fin en soi mais plutôt un moyen de permettre à ce type de pouvoir décentralisé d'intégrer les principes de la modernité telle qu'elle est véhiculée par le projet politique du New Labour. Celui-ci affiche clairement sa volonté non seulement de reléguer aux oubliettes le socialisme municipal ainsi que l'ensemble de son outil de gestion des années 80 - années de divisions au sein du parti -, mais également d'entériner la rupture par rapport à la position travailliste traditionnelle vis-à-vis des collectivités territoriales. Dans le Livre blanc on parle d'une Greater London Authority qui doit être, "stratégique, démocratique, inclusive, efficace, petite, audible et consensuelle " et avoir "une idée claire de son rôle et de l'influence qu'elle pourra exercer ». Dans un article publié dans le Municipal Journal en mars 1998, et qui accompagne ce Livre blanc, Blair réitère ces propos lorsqu'il déclare dans un langage mâtiné de la nouvelle rhétorique de son parti :

Alors que dans chaque localité de nombreux organismes se bousculent pour trouver leur place, les conseils [municipaux et généraux] ont l'occasion de profiter de leur statut unique d'instances élues directement au suffrage universel pour attirer toute une gamme d'autres acteurs vers des partenariats cohérents et productifs ${ }^{11}$.

Et plus loin :

Nous avons besoin d'instances dirigeantes de proximité qui puissent clairement montrer aux métropoles, aux villes et aux villages dans toute la Grande-Bretagne le chemin à suivre et les responsabilités à assumer ${ }^{12}$.

10 Le choix d'établir une mairie à Londres fait l'objet d'un référendum en 1998 et le « oui » l'emporte même si le taux d'abstention est considérable (65\%). Il s'agit à la fois de transférer certaines responsabilités du gouvernement central vers cette nouvelle instance et de concentrer ou rassembler sous le même toit des pouvoirs qui s'étaient éparpillés depuis 1986. Mais si décentralisation il doit y avoir, le New Labour, contrairement à ce qu'il avait pu laisser croire, entend bien garder la mainmise sur la capitale.

\section{« Turn again Livingstone »}

11 Les ambitions du New Labour ne tiennent pas suffisamment compte de Kenneth Livingstone. $\mathrm{Si}$, au début, celui-ci avait déclaré publiquement ne pas être intéressé par le poste de maire de Londres, les sondages le font changer d'avis dans la mesure où on $\mathrm{y}$ découvre qu'il peut représenter une alternative, au moins dans la capitale, à la domination qu'exerce le Premier ministre sur le Parti travailliste ${ }^{13}$. Pour de nombreux Londoniens, leur mairie doit être autre chose qu'une filiale de Millbank, sous le joug du Premier ministre lui-même. Cette envie de tout contrôler depuis le centre ${ }^{14}$ sera dénoncée plus tard par Livingstone dans les toutes premières lignes de son manifeste lorsqu'il brigue son premier mandat de maire :

L'élection d'un maire et d'une assemblée pour Londres permettra de nouveau aux Londoniens de gérer leurs propres affaires et définir leurs propres priorités. Je suis candidat indépendant car je crois que le travail du maire sera de défendre les intérêts de Londres. Si les candidats et les choix politiques peuvent être imposés par le gouvernement central, alors la décentralisation s'en trouvera vidée de sens ${ }^{15}$.

Il faut dire que le New Labour multiplie ses efforts pour écarter Livingstone de la course, au bénéfice de son candidat "officiel» en la personne de Frank Dobson. Lors des 
entretiens avec le comité de sélection, on demande à Livingstone d'approuver le manifeste du parti pour Londres (A Mayor and Assembly for London: the Government's Proposals for Modernising the Governance of London) où, pour rénover le métro vieillissant, il est question de créer des partenariats entre le secteur public et le secteur privé. Livingstone n'en a cure et pour contrer les apparatchiks du New Labour, cite le manifeste de 1997 où les partenariats entre les secteurs public et privé correspondent à un engagement idéologique par rapport à l'ensemble des services publics :

Le Parti travailliste compte instaurer un partenariat public/privé non seulement pour améliorer le métro de Londres mais également pour honorer son engagement envers tout ce qui concerne l'intérêt du public et garantir le meilleur rapport qualité/prix aux contribuables et aux usagers ${ }^{16}$.

13 Selon Livingstone, les intérêts de la capitale ne coïncident pas forcément avec les choix politiques du New Labour. A cet égard la décentralisation ne doit pas se résumer à l'imposition, depuis le centre, des stratégies du parti. L'enjeu, selon lui, est de faire en sorte que la mairie de Londres soit un symbole fort, et qu'elle encourage le gouvernement à organiser davantage de décentralisation ${ }^{17}$. Dans le même article ${ }^{18}$, Livingstone prétend que celle-ci peut constituer l'un des éléments clés de l'avenir du « socialisme ». Tout en versant dans le jargon de la troisième voie, il lance un clin d'œil très complice en direction du Old Labour :

Les socialistes commencent à penser à leur orientation future et il paraît clair que le socialisme devra se préoccuper de la «dévolution", la démocratie et la décentralisation pour offrir aux administrés la possibilité de prendre une part active aux décisions qui les concernent ${ }^{19}$.

De toute évidence Livingstone ne pliera pas l'échine mais par delà sa propension à renouer avec des pratiques et une rhétorique politiques bannies par le New Labour, il dénonce aussi le chemin que prend "son " parti vers la commercialisation pure et simple des services publics ${ }^{20}$. D'autre part, la création d'une mairie à Londres ne doit pas être, selon lui, l'occasion pour le New Labour de disposer d'un relais de plus pour diffuser le message du parti. C'est ainsi que Livingstone entame bel et bien sa nouvelle carrière de franc-tireur et compromet sérieusement ses chances de recevoir l'investiture du New Labour. Sa défiance risque, aussi, de lui valoir d'en être exclu.

Pour choisir son candidat, le New Labour organise ce qu'il convient d'appeler un simulacre d'élection ${ }^{21}$. Il constitue un collège électoral et dispose également des votes "bloqués" des syndicats ${ }^{22}$. Frank Dobson sort victorieux de cette élection, mais Livingstone n'accepte pas le résultat qui porte manifestement la marque du control freakery orchestré par le cabinet du Premier ministre. Après quelques jours d'hésitation, Livingstone annonce sa candidature en tant que candidat indépendant :

J'ai été obligé de choisir entre le parti que j'aime et faire respecter les droits démocratiques des Londoniens. J'en ai conclu que pour défendre le principe selon lequel la ville de Londres a le droit de se gouverner elle-même, je me dois de me porter candidat indépendant aux élections pour choisir le maire de Londres le 4 $\mathrm{mai}^{23}$.

La sanction ne tarde pas à tomber et, comme prévu, il est exclu du parti pour cinq ans.

\section{L'élection de 2000}

17 On assiste ensuite à une campagne où, compte tenu du climat politique, les projets des uns et des autres sont rapidement et très largement relégués au deuxième plan. 
L'omniprésence des médias contribue à la personnalisation à outrance de la campagne, où les petites phrases assassines visent directement les différents candidats. Livingstone, bien plus à l'aise sous les feux de la rampe que ses concurrents, joue allègrement de son statut de " victime » de la machine électorale travailliste, et réussit à s'attirer une certaine sympathie - voire solidarité - de la part du public. Alors que Dobson met en avant l'orthodoxie budgétaire du New Labour, Livingstone admet volontiers la possibilité de faire appel au secteur privé pour faire fonctionner un service public, et notamment son propre sujet de prédilection, les transports en commun. Ceux-ci souffrent depuis longtemps d'un manque chronique d'investissements et l'idée du New Labour, pour rénover l'infrastructure, consiste à créer des partenariats hybrides qui impliqueront à la fois les secteurs public et privé24.

Livingstone a l'impression, cependant, que sur ce dossier précis le New Labour cède trop aveuglément aux sirènes du néo-libéralisme en privilégiant la marchandisation d'un service public au rôle majeur dans la capitale. À tel point qu'il estime, toujours par le truchement de la presse, que la question de la rénovation du métro, et par la suite de toute l'infrastructure des transports en commun, permettra à l'électorat londonien de mesurer le degré d'autonomie qui sera accordé à la mairie. Cette question devient rapidement le cheval ${ }^{25}$ de bataille électoral de Livingstone. Selon lui, choisir le candidat du New Labour revient forcément à accepter une emprise forte de la politique nationale, au détriment à la fois des services publics dans la capitale et de l'autonomie qui doit accompagner la décentralisation.

Le 4 mai 2000 Livingstone est déclaré vainqueur de la course à la mairie de Londres ${ }^{26}$. Plusieurs raisons peuvent expliquer cette victoire et également l'humiliation du candidat officiel du New Labour, classé en troisième position juste devant le candidat des libéraux démocrates. Il paraît clair que cette élection était l'occasion rêvée pour les Londoniens de faire un pied de nez à Blair et à toute sa machine électorale, celle qui avait truqué le processus de sélection du candidat officiel. On ne peut pas non plus sous-estimer l'envie de ce même électorat de dénoncer l'arrogance du New Labour et le contrôle excessif qu'il semble vouloir exercer à tous les niveaux du parti. Malgré sa volonté affichée de favoriser la représentation politique de proximité, le New Labour avait montré ses réels objectifs, dans cette course, en matière de décentralisation. À tel point que de nombreux électeurs avaient clairement annoncé leur intention de ne pas voter pour son candidat « officiel », quel qu'il soit ${ }^{27}$.

Dans un article publié en mars 2000 dans la Local Government Chronicle, un membre du New Labour, Francis Beckett, explique que «les subterfuges utilisés par le New Labour et l'obsession qu'il a de tout contrôler obligeront de nombreux fidèles à changer de camp». On peut se contenter de penser que «l'autre camp » signifie ici celui de Livingstone. Mais la désaffection pour le New Labour est si profonde que tout porte à croire que pour certains sympathisants, même l'alternative offerte par le candidat indépendant ne constitue pas une protestation suffisante. D'où l'envie, peut-être, pour l'électorat londonien, d'avoir un vrai franc-tireur à la tête de l'assemblée de Londres plutôt qu'un homme de main de Blair. Les Londoniens espèrent, sans doute, que le nouveau maire saura contrebalancer le pouvoir politique central et qu'il n'aura pas peur d'imposer l'intérêt de la ville plutôt que systématiquement celui du parti. Au lendemain de l'élection, le ressentiment éprouvé par l'électorat londonien est étalé en bonne et due forme dans toute la presse. Hormis l'avalanche d'opinions purement partisanes, on y apprend que le New Labour s'est fondamentalement trompé en pensant pouvoir imposer 
son choix à Londres mais surtout en essayant de faire taire l'un des défenseurs des valeurs associées au Old Labour. Pour calmer les esprits, l'équipe du Premier ministre déclare vouloir mettre un terme au système d'investiture du parti, mais le mal est fait. Le temps de cette élection, Livingstone est devenu la coqueluche des Londoniens mais le sentiment général est que dorénavant, il pourra être jugé selon ses actes.

\section{Le premier mandat : une affaire de transportsen commun}

21 Le maire de Londres est responsable du groupe de la Greater London Authority, qui comprend l'assemblée elle-même, Transport for London, la London Development Agency chargée du développement économique de la capitale et notamment de l'urbanisme, la Metropolitain Police Authority sous la tutelle du ministre de l'Intérieur, et la London Fire and Emergency Planning Authority placée sous la tutelle du ministre des Transports. Les décisions budgétaires relatives à ces deux derniers organismes relèvent, en ce qui concerne la capitale, à la fois de la responsabilité du maire et des ministères concernés. La GLA est composée de 25 membres élus en même temps que le maire. Quatorze de ces membres sont élus par les Londoniens selon un découpage géographique de la capitale. Les onze autres sont élus par le biais d'un système proportionnel et sont connus sous le nom de Londonwide Members. La tâche principale des membres de l'assemblée consiste à suivre de près les décisions prises par le maire, et de lui demander des comptes. Puisque l'assemblée n'a aucun pouvoir pour s'opposer à la volonté du maire, seul le sort des urnes, tous les quatre ans, peut l'écarter de son siège de responsable exécutif de la GLA. Le budget dont dispose la mairie est constitué à la fois d'un pourcentage "GLA » de l'impôt local, et de la subvention octroyée par le gouvernement central. La GLA peut également trouver des sources de financement propres, l'exemple le plus connu étant bien entendu la taxe anti-embouteillages, dont les bénéfices sont réinjectés dans les transports en commun ${ }^{28}$. De l'aveu général, ces derniers constituent "la définition même du mandat de maire de $M$. Livingstone ${ }^{29}$. Dès sa prise de fonctions, le maire croise le fer avec le gouvernement au sujet du mode de financement de la rénovation et modernisation du métro. Alors que Livingstone avait préconisé, pendant sa campagne, la création d'un actionnariat populaire pour améliorer le métro ${ }^{30}$, le New Labour continue de vanter les mérites du partenariat public/privé (PPP), l'idée étant de faire appel au secteur privé pour venir en aide au secteur public. Livingstone n'est pas fondamentalement opposé au principe du PPP et réitère son intention d'utiliser les compétences là où elles se trouvent. Robert Kiley, celui qui a su brillamment rénover les transports en commun de New York, est nommé directeur de Transport for London. Son contrat juteux fait grand bruit, d'autant que Livingstone avait toujours été prompt à dénoncer les "fat-cat salaries " perçus par certains dirigeants de services publics. Mais si le socialisme municipal du GLC semble avoir connu quelques mutations depuis ses heures de gloire d'avant 1986, jusqu'à l'intégration de quelques menus principes du libéralisme, la combativité de Livingstone reste intacte. Le maire ne veut pas voir la capitale acculée à accepter une gestion du métro choisie et mise en place par le gouvernement central. Sa réticence est d'autant plus forte que la capitale sera contractuellement liée aux entreprises impliquées - Metronet et Tubelines en l'occurrence - pour une période de trente ans. Outre le fait que le maire veut faire valoir l'indépendance de la mairie en ce qui concerne les choix stratégiques pour la 
capitale, ce sont surtout des problèmes de sécurité, à la suite de la catastrophe de Hatfield ${ }^{31}$, qui expliquent sa réticence quant au rôle que peut jouer le secteur privé dans les transports.

Le conflit prend rapidement la forme d'un "débat de société » car le maire dispose, dorénavant, d'une tribune - et surtout d'un budget - légitimes pour s'en expliquer auprès $d u$ public. La rénovation du métro met en lumière deux des principes fondamentaux de l'idéologie du New Labour: d'une part il est inconcevable qu'une administration publique puisse emprunter des fonds en son nom propre, ce qui de fait condamne l'idée de créer un actionnariat populaire. D'autre part, le secteur privé est de toutes les manières plus efficace que le secteur public. Dans un premier temps les négociations entre le gouvernement et Robert Kiley au sujet du PPP sont cordiales. Ils semblent être sur la voie d'un compromis qui écartera, au moins, la fragmentation de la gestion du métro entre une pléthore d'entreprises privées et Transport for London. En mars 2001, la situation se gâte pourtant, et Kiley, avec le soutien de Livingstone qui pense avoir été élu, justement, pour s'opposer aux projets tels que le PPP, porte l'affaire devant les tribunaux. Selon Livingstone : "La décision du gouvernement d'essayer d'imposer le PPP à la capitale montre son mépris pour l'opinion d'une majorité écrasante de Londoniens ॥". Le PPP est rebaptisé partial privatisation plan par le maire qui souligne le trou budgétaire que la ville va devoir combler lorsque la responsabilité pour le métro sera transférée de London Underground à Transport for London ${ }^{33}$. Mais le 31 juillet 2001, en dépit du fiasco financier autour de l'extension de la Jubilee Line, Livingstone est débouté par le tribunal. Le juge déclare qu'en vertu du Greater London Act de 1999, le gouvernement central est tenu de décider «en tenant compte » et non pas «en fonction » de l'avis du maire. La Cour européenne est saisie, mais en juillet 2002 après une coûteuse campagne - quelque quatre millions de livres d'argent public - Livingstone et Kiley renoncent à contester le PPP. Il s'agira maintenant, selon leurs propres termes, de négocier les meilleures conditions pour la ville en fonction du PPP proposé.

\section{L'élection de 2004. Livingstone : renégat ou candidat?} 34

Vers la fin de 2003, la presse étale au grand jour le retour possible de Livingstone au sein de sa famille politique. Les manchettes font suite au nombre croissant de manœuvres entreprises par le Parti travailliste pour réintégrer son enfant terrible. Le New Labour a beau jeu d'expliquer que cette fois-ci Livingstone sera jugé à l'aune du travail accompli à City Hall, et le Premier ministre lui-même déclare que "[p]our être honnête, il a fait du bon boulot $»^{35}$. L'année précédente, pourtant, le refus prononcé au mois de juillet par le NEC de ne pas surseoir à l'exclusion de Livingstone, avait semblé sans appel. A cet égard, il est difficile de croire que la volte-face effectuée par le New Labour est exclusivement liée au bilan du premier mandat du maire. Tout d'abord, le New Labour n'est pas en mesure de proposer une candidature qui puisse rivaliser avec celle de Livingstone ${ }^{36}$. Celui-ci souhaite réintégrer le Parti travailliste mais dit clairement qu'il n'hésitera pas à être de nouveau candidat indépendant si son parti ne veut pas lui accorder son investiture. Mais par-dessus tout, le cabinet du Premier ministre craint les répercussions potentielles d'un deuxième fiasco à la mairie de Londres sur les élections européennes et municipales en Angleterre et au Pays de Galles, prévues le même jour. Sans oublier l'imminence d'élections législatives qui 
seront probablement organisées en 2005. Par ailleurs, si le New Labour sait que Livingstone a de fortes chances de remporter cette élection, les travaillistes, à défaut d'obtenir le siège de maire, veulent tout de même maintenir leur influence à la mairie de Londres en termes d'élus à l'assemblée. Une deuxième élection ratée risquerait non seulement d'affaiblir la crédibilité du New Labour, incapable de gérer la popularité de Livingstone, mais également de porter un coup à ses projets de décentralisation, compte tenu des scrutins régionaux prévus pour l'automne ${ }^{37}$.

Cependant, pour que Livingstone soit le candidat officiel du New Labour, il doit d'abord être membre du parti et a fortiori, le soutenir. Interrogé sur cette question, Livingstone nie avoir trahi son parti et met en avant, une fois de plus, le manifeste électoral travailliste de 1997 où, selon lui, la défense des services publics est l'un des principaux engagements du Parti. Or, même si la mairie de Londres n'a pas réussi à faire obstruction au PPP proposé pour le métro, le maire entend bien, s'il doit défendre les couleurs travaillistes, pouvoir intervenir sur les engagements futurs de son parti pour Londres.

Le 25 novembre 2003, Livingstone est déclaré par la Political Studies Association " personnage politique de l'année ", en vertu de son "courage exemplaire lors de l'introduction de la taxe anti-embouteillages ». Cette nomination fait suite à un sondage qui a permis, au mois de septembre 2003, de lui décerner le titre de "personnage ayant le plus d'autorité dans les services publics en Grande-Bretagne ». Compte tenu de ces éloges, il semblerait que le NEC ait surtout fait preuve de pragmatisme - what counts is what works -, lorsque le 6 janvier 2004, après un bref "test de loyauté ", la décision est prise de réadmettre Livingstone au sein du parti. Cette décision est confirmée le 2 février lors d'un vote des adhérents londoniens du Parti travailliste. Pour sauver la face, le New Labour insiste, dans la presse, sur le fait que le maire « candidat officiel du parti » devra se conformer au règlement. Très enclin à des commentaires à l'emporte-pièce sur divers sujets n'ayant rien à voir avec son mandat de maire, Livingstone va devoir rentrer dans le rang ${ }^{38}$.

Le maire n'est pas dupe et verse allègrement dans l'ironie : «Si le parti veut me reprendre, ce n'est pas à cause de mon charme personnel, mais pour tirer profit de mon bilan ${ }^{39}$. Même s'il ne chante pas victoire, Livingstone ne manque pas de pavoiser : "Ces deux dernières décennies ont été dominées par deux personnages politiques: Blair et Thatcher. Les deux ont essayé de m'anéantir et les deux ont échoué " ${ }^{40}$. Il sait que son statut de candidat "renégat " lui confère l'indépendance nécessaire, pendant les six mois avant les élections, pour rendre la mairie de Londres encore plus crédible en tant que pouvoir décentralisé. Par ailleurs, s'il est disposé à superviser la mise en place du PPP qu'il a combattu avec autant de véhémence, il entend bien tirer profit de son nouveau statut de «candidat officiel ». Celui-ci n'est pas la manifestation de sa collusion avec le New Labour mais plutôt de sa volonté de créer une certaine synergie entre les deux pouvoirs, central et supra-muncipal. Pour ce faire, Livingstone exige un rôle majeur dans l'élaboration du manifeste, ce qui lui est accordé le jour où sa candidature reçoit l'aval du Parti travailliste de Londres.

À la suite de sa réintégration, la cote de popularité de Livingstone baisse car on s'interroge sur le « coût » d'une telle manœuvre. Saura-t-il continuer, depuis l'intérieur du parti, à défendre les intérêts de la capitale? La mesure phare de son premier mandat, la taxe anti-embouteillages est celle qui assure sa crédibilité politique personnelle en tant que maire. Si bien que Livingstone caresse l'idée d'agrandir la zone 
concernée, au grand dam du candidat conservateur qui promet de l'abolir s'il est élu en juin 2004. Une des conséquences de la taxe est l'augmentation spectaculaire du nombre de trajets effectués dans la capitale en bus, un domaine où la mairie a mené des actions de communication et de financement très en vue. Des contrats qui garantissent un service de qualité et qui comprennent des sanctions financières sont la fierté du maire, scandalisé par l'attitude désinvolte des entreprises privées qui assurent la gestion des transports ${ }^{41}$. À terme, la mairie souhaiterait assumer la responsabilité des chemins de fers londoniens, de manière à lui permettre d'élaborer une stratégie des transports en commun pour l'ensemble de la capitale. L'effet « olympique » est ici fortement ressenti.

Compte tenu de la nouvelle affiliation politique de Livingstone, on aurait pu s'attendre à ce que son manifeste porte la marque ostensible du New Labour. Or, il n'en est rien et les allusions au parti sont assez rares pour être invisibles; comme si une fois l'appartenance politique du maire confirmée, il a cherché à s'affranchir publiquement $\mathrm{du}$ gouvernement central. Pourtant, Blair et Livingstone partagent, tous les deux, un goût exacerbé pour le pragmatisme, le populisme et les principes. Compte tenu de cette similitude, la création de la mairie de Londres aurait pu les mettre sur la même voie mais pas nécessairement la troisième. Force est de constater que la confrontation entre Livingstone et l'équipe politique du Premier ministre alimente, au contraire, le débat sur les valeurs fondamentales communément associées - à tort ou à raison - au Old et au New Labour.

\section{Conclusion}

29 Le procès - perdu d'avance - entre la mairie de Londres et le gouvernement de Blair au sujet du PPP proposé pour le métro a mobilisé les foules. Celle massée devant les portes du tribunal et acclamant Ken Livingstone témoigne, d'une certaine façon, de l'attachement des Britanniques aux valeurs collectivistes que d'aucuns voudraient voir définitivement enterrées. Cette proclamation de soutien pour Livingstone a presque fait oublier le coût exorbitant du procès, financé par l'argent public, ainsi que le retard considérable subi par le projet de rénovation du métro. En même temps, cette contestation souligne la possibilité, à l'échelle locale, de remettre en question des décisions émanant directement de Westminster. Si le modèle économique du New Labour impose des choix, même déguisés et présentés sous forme de "partenariats", troisième voie oblige -, la Mairie de Londres n'accepte pas l'idée selon laquelle libéralisme et maintien de services publics de qualité seraient incompatibles.

C'est essentiellement pour cette raison que dans son projet de décentralisation, l'autonomie proposée à la mairie de Londres par le New Labour s'est avérée insuffisante. Un maire issu du sérail s'en serait sans doute contenté et dans ce cas, son rôle aurait pu se limiter à celui d'un simple relais logistique pour le gouvernement central. Livingstone, en revanche, pour exercer convenablement le mandat pour lequel il a été élu, s'est vu rapidement contraint de contester cette autonomie et d'exiger plus d'indépendance. Ce faisant, il a créé un espace politique dans lequel peuvent se retrouver ceux qui ont du mal à admettre le manque de rupture entre le New Labour et ses prédécesseurs conservateurs ${ }^{42}$; ceux qui pensent que la conquête par Livingstone de la mairie de Londres n'est pas une simple anecdote du paysage politique britannique. De ce point de vue, il y a fort à parier que le maire de Londres est bien plus 
en adéquation avec les espoirs de la victoire électorale de 1997 qu'avec les nombreuses déceptions intervenues depuis.

\section{BIBLIOGRAPHIE}

DEPARTMENT OF THE ENVIRONMENT, TRANSPORT AND THE REGIONS. A Mayor and Assembly for London : the Government's Proposals for Modernising the Governance of London. London: Stationery Office, Cmnd. 3897, October 2002.

CARVEL, John. Turn Again Livingstone. London: Profile Books, 1999.

CROUCH, Colin. A Threat to Citizenship : The Commercialisation of Public Services. Florence, European University Institute, March 2003.

FLYNN, Norman ; LEACH, Steve \& VIELBA, Carol. Abolition or Reform : the GLC and the Metropolitan County Councils. London : Allen \& Unwin, 1985.

FORRESTER, Andrew ; LANSLEY, Stewart \& PAULEY, Robin. Beyond our Ken. London: Fourth Estate, 1985.

LEYDIER, Gilles (ed.). Les Services publics britanniques. Presses Universitaires de Rennes, 2004.

SOFER, Anne. The London Left Takeover. London: Jaguar Press, 1987.

Streamlining the Cities, Cmnd. 9063. October 1983.

WHITTON, Timothy (ed.). Le New Labour : rupture ou continuité ? Presses Universitaires de Rennes, 2000.

\section{NOTES}

1. Appelée congestion charge.

2. Le nom du nouveau bâtiment qui abrite la mairie de Londres.

3. Pour plus de précisions concernant cette période de l'histoire du Conseil du Grand Londres voir mon article «La Mairie de Londres : l'exception qui confirme la règle » in Gilles LEYDIER (ed.), Les Services publics britanniques, Presses Universitaires de Rennes, 2004, pp. 257-274.

4. Il y a fort à parier que la volonté d'écarter Livingstone avant que ses choix politiques ne puissent trop nuire, selon les conservateurs, à Londres, a donné naissance au mythe de « Ken le Rouge » et contribué à sa grande notoriété - et parfois popularité - publiques.

5. County Hall fut vendu en 1992 à la Shirayama Corporation pour 65 millions de livres. Le bâtiment accueille actuellement l'aquarium de Londres, un hôtel, 700 appartements de luxe et même un restaurant McDonald's.

6. Dès sa prise de pouvoir en mai 1981, Livingstone déclare vouloir se servir de son mandat pour combattre le gouvernement conservateur.

7. Les autres Conseils Métropolitains sont : Greater Manchester, Merseyside, South Yorkshire, Tyne and Wear, West Midlands et West Yorkshire.

8. Les élections de 2000, du moins le volet qui concerne l'investiture du candidat du New Labour, ont été tout sauf propres. Rétrospectivement cette rubrique porte mal son titre. 
9. Local decision-making should be less constrained by central government, and also more accountable to local people. [...] We will encourage democratic innovations in local government, including pilots of the idea of elected mayors with executive powers in cities.

10. C'est moi qui souligne. London is the only Western capital without an elected government. Following a referendum to confirm popular demand, there will be a new deal for London, with a strategic authority and a mayor, each directly elected. Both will speak up for the needs of the city and plan its future. They will not duplicate the work of the boroughs, but take responsibility for London-wide issues - economic regeneration, planning, policing, transport and environmental protection. London-wide responsibility for its own government is urgently required.

11. With many local-level organisations jostling for position, councils have the opportunity to use their unique role as directly elected bodies to draw the disparate range of other players into coherent and working partnerships.

12. We need strong local leadership that gives clear direction and accountability to cities, towns and villages over Britain.

13. L'un des anagrammes de « Ken Livingstone Mayor of London » est : I'll soon annoy government, kid!

14. Control freakery.

15. The direct election of a Mayor and Assembly for London will give Londoners back the right to govern their own affairs and decide upon their own priorities. I am standing as an independent candidate because I believe the job of the Mayor will be to stand up for London. If candidates and policies can be imposed centrally then devolution will mean nothing.

16. C'est moi qui souligne. Labour plans a new public/private partnership to improve the Underground, safeguard its commitment to public interest and guarantee value for money to taxpayers and passengers.

17. Même si le récent résultat catastrophique - $78 \%$ de «non » contre $22 \%$ de « oui »- lors du référendum concernant une assemblée régionale pour le Nord-Est a poussé John Prescott à annuler les référendums prévus dans les autres régions, le 'wider programme of devolution' du New Labour est maintenu. The Guardian, 9 November 2004, p. 10.

18. Local Government Chronicle, 20/04/2000.

19. Socialists are beginning to rethink what is the wave of the future, and clearly socialism has to be about devolution, democracy, decentralisation and empowering local people.

20. Colin CROUCH, A Threat to Citizenship : the Commercialisation of Public Services, European University Institute, Florence, March 2003.

21. Voir note 3.

22. Le New Labour s'est servi des votes «bloqués » des syndicats pour imposer «son » candidat, Alun Michael, à la tête de l'assemblée au Pays de Galles à la place du populaire Rhodri Morgan. Pourtant, le New Labour reproche souvent à l'ancien parti old Labour d'avoir abusé de cette méthode pour élire Kinnock, Hattersley, Smith et ... Blair, même si c'est l'élection, en 1981, de Healey aux dépens de Benn qui reste gravée dans les esprits.

23. C'est moi qui souligne. I have been forced to choose between the party I love and upholding the democratic rights of Londoners. I have concluded that defence of the principle of London's right to govern itself requires that I stand as an independent candidate for London mayor on 4 May. 'Livingstone: I'm standing', The Guardian, 6 March 2000.

24. Appelés Public Private Partnerships (PPP).

25. Ou plutôt « bus » puisque pendant sa campagne, Livingstone sillonne les rues de Londres dans un bus mauve orné de son slogan Ken4London.

26. Taux de participation : 33,65\%. Lors du premier tour Livingstone obtient 38,96\% des voix, Steven Norris le candidat conservateur 27,09\%, Frank Dobson (New Labour) 13,06\%, Susan Kramer (LD) $11,87 \%$. Au deuxième tour, Livingstone obtient $57,92 \%$ des voix et son rival, Steven Norris, $42,08 \%$. 
27. Ceci peut expliquer la consternation des Londoniens après les élections lorsqu'ils apprennent que le New Labour envisage de réintégrer Livingstone. On craint que son indépendance vis-à-vis du pouvoir central ne soit compromise par cette nouvelle affiliation.

28. Pour plus de renseignements, consulter le site de la mairie de Londres, < http:// www.london.gov.uk/>, ou bien <http://www.citymayors.com/uk/london.html>.

29. 10/01/04, p. 9.

30. L'objectif de cet actionnariat populaire est de générer les finances nécessaires à la rénovation du métro, tout en impliquant les usagers dans le projet. L'idée de Livingstone est d'encourager les usagers à investir dans le métro en leur proposant d'acheter des titres boursiers.

31. Le 17 octobre 2000, quatre passagers sont tués lors d'un accident de train à Hatfield. La commission d'enquête relève des défaillances mécaniques importantes et on soupçonne Balfour Beatty, l'entreprise privée responsable de cette partie des rails, d'avoir négligé l'entretien.

32. The government's decision to try to impose the PPP on the capital is to show contempt for the overwhelming views of London.

33. Ce transfert devient effectif le 15 juillet 2003.

34. Le site suivant propose un descriptif des candidats ainsi que leurs manifestes électoraux: <http://www.londonelects.org.uk/candidates/mayor/livingstone.html>.

35. Ce qui compte est ce qui marche. What counts is what works.

36. Nicky Gavron, la première adjointe au maire, est la candidate " officielle » du New Labour depuis 2002.

37. Voir note 17.

38. Parmi ses déclarations tonitruantes on se souviendra de celle où il appela publiquement George Bush, en visite d'État en Grande-Bretagne, "l'homme le plus dangereux de la planète ». Plus récemment Livingstone a déclaré vouloir voir le premier ministre israélien en prison et la famille royale saoudienne "envoyée à la lanterne».

39. The reason the Labour party wants me back is because they want to run on my record. It is not my personal charm. Hugh MUIR, 'Blair and Thatcher tried to crush me. Both failed', The Guardian, 31 May 2004.

40. There have been two dominant figures of the last two decades. Blair and Thatcher. Both have tried to crush me and both have failed. Ibid.

41. A partir de 1985, les opérateurs privés pouvaient soumissionner pour exploiter des lignes de bus avant la privatisation officielle qui devint effective en 1994. Le réseau ferré de banlieue fut privatisé en même temps que les lignes nationales, pour l'essentiel, entre 1993 et 1997.

42. Timothy WHITTON (ed.), Le New Labour: rupture ou continuité ?, Presses Universitaires de Rennes, 2000.

\section{RÉSUMÉS}

Dans son manifeste électoral de 1992 le Parti travailliste envisage de rétablir une autorité municipale à Londres. La décentralisation vers une instance « supra » municipale dans la capitale s'inscrit dans son programme global de modernisation, leit motiv, par excellence, de la troisième voie. C'était sans tenir compte de Kenneth «le Rouge» Livingstone, défenseur acharné de certaines valeurs collectivistes chères au old Labour que Blair et les siens voudraient avoir définitivement reléguées aux oubliettes du parti. Fort de sa popularité d'abord comme candidat, 
puis comme maire élu, Livingstone entend bien faire en sorte que la mairie de Londres ne passe pas sous les fourches caudines du gouvernement central. Dans un premier temps, fidèle à ses habitudes de l'époque du Greater London Council, Livingstone incarne le contre-pouvoir. Sa deuxième victoire en juin 2004, en revanche, sera l'occasion pour l'électorat londonien de juger le maire et la mairie de Londres selon leur bilan.

In its 1992 manifesto the Labour Party considered setting up a municipal authority in London. Empowering a pan-London authority was part and parcel of Labour's global programme of modernisation, one of the outstanding leitmotifs of the third way. But these plans did not take Kenneth Livingstone sufficiently into account. "Red" Ken still championed some of the collectivist values cherished by Old Labour which Blair and his friends would like to have once and for all consigned to the history of the Labour party. Taking full advantage of his popularity as a candidate and then as elected mayor, Livingstone was determined to make sure that the Greater London Authority should not be subjected to the will and whims of central government. At the outset, remaining faithful to his Greater London Council ways, Livingstone personified opposition to central government. His second victory in June 2004, on the other hand, was the opportunity for Londoners to judge their mayor and municipal authority according to their track record.

\section{AUTEUR}

\section{TIMOTHY WHITTON}

Université Blaise Pascal, Clermont-Ferrand II 